Article

\title{
Photoinduced Metal-Free Surface Initiated ATRP from Hollow Spheres Surface
}

\author{
Chun-Na Yan ${ }^{1,+}$, Qian Liu ${ }^{1,+}$, Lin Xu ${ }^{2}$, Li-Ping Bai ${ }^{1}$, Li-Ping Wang ${ }^{1, *}$ and Guang $\mathrm{Li}^{1}{ }^{1} *$ (D) \\ 1 College of Materials Science and Engineering, Liaocheng University, Liaocheng 252059, China; \\ YCN5053@163.com (C.-N.Y.); liuqianbang@163.com (Q.L.); blp9641@163.com (L.-P.B.) \\ 2 College of Materials Science and Engineering, Qingdao University, Qingdao 266071, China; \\ xulinbang@163.com \\ * $\quad$ Correspondence: wangliping5@163.com (L.-P.W.); lglzsd@126.com (G.L.); Tel.: +86-635-8230-919 (L.-P.W.) \\ + These authors contributed equally to this work.
}

Received: 15 March 2019; Accepted: 26 March 2019; Published: 2 April 2019

check for updates

\begin{abstract}
Well-defined amphiphilic diblock copolymer poly (methyl methacrylate)-b-poly (N-isopropylacrylamide) grafted hollow spheres (HS-g-PMMA- $b$-PNIPAM) hybrid materials were synthesized via metal-free surface-initiated atom transfer radical polymerization (SI-ATRP). The ATRP initiators $\alpha$-Bromoisobutyryl bromide (BIBB) were attached onto hollow sphere surfaces through esterification of acyl bromide groups and hydroxyl groups. The synthetic ATRP initiators (HS-Br) were further used for the metal-free SI-ATRP of methyl methacrylate (MMA) and N-isopropyl acrylamide (NIPAM) using 10-phenylphenothiazine (PTH) as the photocatalyst. The molecular weight of the polymers, structure, morphology, and thermal stability of the hybrid materials were characterized via gel permeation chromatography (GPC), X-ray photoelectron spectroscopy (XPS), ${ }^{1} \mathrm{H}$-nuclear magnetic resonance spectroscopy $\left({ }^{1} \mathrm{H}\right.$ NMR), transmission electron microscopy (TEM), Fourier transform infrared spectroscopy (FT-IR), and thermogravimetric analysis (TGA), respectively. The results indicated that the ATRP initiator had been immobilized onto HS surfaces successfully followed by metal-free SI-ATRP of MMA and NIPAM, the Br atom had located at the end of the main PMMA polymer chain, and the polymerization process possessed the characteristic of controlled/"living" polymerization. The thermal stability of the hybrid materials was increased significantly compared to the pure PMMA and PNIPAM.
\end{abstract}

Keywords: hollow spheres; metal-free ATRP; SI-ATRP; 10-phenylphenothiazine (PTH); thermal stability

\section{Introduction}

Surface-initiated radical polymerization is an important synthetic method for the preparation of polymer-inorganic hybrid materials [1-5]. Different "living" radical polymerization methods, such as atom transfer radical polymerization (ATRP) [6-8], reversible addition-fragmentation chain transfer (RAFT) [9-11], and reverse iodine transfer polymerization (RITP) [12-14], have been used to modify inorganic material surfaces with controlled polymer chain length, composition, and architecture via surface-initiated radical polymerization methods. Atom transfer radical polymerization (ATRP) is one of the most versatile and robust methods for synthesis of polymers with controlled molecular weight (MW), molecular weight distribution (MWD), structure, and functionality $[15,16]$. Surface-initiated ATRP has been successfully used to graft polymers from a variety of solid surfaces of different shapes and materials, such as $\alpha-\mathrm{Fe}_{2} \mathrm{O}_{3}$ rods [17], iron oxide magnetic nanoparticles [18], SBA-15 [19], wood flour [20], and so on. However, ATRP is also practically discouraged due to the disadvantage of using ATRP metal catalysts, such as $\mathrm{Cu}^{+}$and $\mathrm{Fe}^{2+}$, which result in the presence of an inevitable metal residue in final products [21-23]. Although successful attempts for various low-ppm catalyst ATRP methods 
have been claimed [24-27], low levels of catalyst remained in the products, which impeded their use, especially in some electronic or biomedical applications [28,29].

In 2014, Treat et al. first reported a metal-free ATRP process, which was mediated by light and catalyzed by an organic-based photoredox catalyst, 10-phenylphenothiazine (PTH) [30]. The new photoinduced metal-free ATRP does not require heavy metal catalysts (such as copper ions and iron ions), so it can avoid the toxicity of metal catalysts. Extensive research has been carried out on photoinduced metal-free ATRP since then. The research group led by Y. Yagci reported a novel synthetic strategy for the syntheses of hyperbranched homo and block copolymers by applying the photoinduced metal-free ATRP [31], and the photoinduced metal-free ATRP activated by highly conjugated electron-rich thienothiophene derivatives was also developed by this group [32]. Wang and co-workers demonstrated metal-free ATRP of biomass-based monomers (e.g., soybean oil, rosin acid, and furfural) under low intensity UV LED light [33]. Matyjaszewski and co-workers further investigated the mechanism of photoinduced metal-free ATRP [34] and expanded the photoredox catalysts using three phenothiazine derivatives as catalysts for photoinduced metal-free ATRP of acrylonitrile [35]. Photoinduced metal-free ATRP was also employed to the surface modification of various materials, such as silica [28], SBA-15 [36], nanodiamond (ND) [37], hydroxyapatite (HAp) nanorods [38], and others.

$\mathrm{SiO}_{2}$ hollow spheres (HS) with a large internal void, hollow cavity, small density, and large specific surface area have received much attention owing to their wide potential applications in adsorption and biomacromolecules delivery [39], electrical materials and catalysis [40], optical performance [41], controlled drug-delivery carriers [42], electrical sensing devices [43], and so on [44,45]. Although pure $\mathrm{SiO}_{2} \mathrm{HS}$ have excellent properties, their applications have been largely restricted due to their poor hydrophobicity and single physical loading ability. Therefore, the surface modification of $\mathrm{SiO}_{2}$ HS with different polymers to improve their dispersibility in various solvents and to extend their physicochemical properties is very essential [46-48].

In the present work, the well-defined HS- $g$-PMMA and HS- $g$-PMMA- $b$-PNIPAM hybrid materials were prepared via metal-free surface-initiated atom transfer radical polymerization (SI-ATRP) for the first time. Firstly, the ATRP initiators $\alpha$-Bromoisobutyryl bromide (BIBB) were attached onto hollow sphere surfaces through esterification of acyl bromide groups and hydroxyl groups. The synthetic ATRP initiators (HS-Br) could be further used for surface polymerization with methyl methacrylate (MMA) and N-Isopropyl acrylamide (NIPAM) monomer using 10-phenylphenothiazine (PTH) as the photocatalyst. The hybrid materials synthesized through metal-free SI-ATRP will broaden the application prospect of ATRP and HS.

\section{Materials and Methods}

\subsection{Materials}

$\mathrm{NaOtBu}$, phenothiazine, RuPhos Precat, and RuPhos were purchased from Sigma-Aldrich (Shanghai, China). $\alpha$-Bromoisobutyryl bromide (BIBB) was acquired from Shanghai Aladdin Chemical Reagent Co. Ltd (Shanghai, China). Methyl methacrylate (MMA), N-Isopropyl acrylamide (NIPAM), and Dimethylacetamide (DMA) were purchased from Shanghai Sinopharm Chemical Reagent Co. Ltd (Shanghai, China). 10-Phenylphenothiazine (PTH) and the hollow spheres were synthesized according to previously described literature, respectively [30,49]. The ${ }^{1} \mathrm{HNMR}$ of PTH can be found in Figure S1. All other chemical reagents were analytical grade and used without further purification.

\subsection{Instrumental Characterization}

The molecular weights and polydispersities (PDI) of the polymers were measured at $30{ }^{\circ} \mathrm{C}$ on a gel permeation chromatographer (GPC) (Waters 1515, Milford, MA, USA) equipped with a 2414 refractive detector. Tetrahydrofuran (THF) was used as an eluent at a flow rate of $0.6 \mathrm{~mL} / \mathrm{min}^{-1}$. Transmission electron microscope (TEM) images were obtained using a JEOL JEM-1400 (JEOL, Tokyo, Japan) transmission electron microscope. The diameter of wall thickness and the average diameter 
of the hollow spheres were calculated using Intel Integrated Performance Primitives (ipp). ${ }^{1} \mathrm{H}$ NMR spectra were recorded on a $400 \mathrm{MHz}$ (Varian Mercury Plus 400, Palo Alto, CA, USA) nuclear magnetic resonance instrument, using $\mathrm{CDCl}_{3}$ as solvent and tetramethylsilane (TMS) as the internal reference. The structures of samples were characterized by Nicolet-100 Fourier (Thermo Fisher Scientific, MA, USA) transform infrared spectroscopy (FT-IR) from $400 \mathrm{~cm}^{-1}$ to $4000 \mathrm{~cm}^{-1}$ using the $\mathrm{KBr}$ pellet method. X-ray photoelectron spectroscopy (XPS) was performed on an Escalab 250xi (Thermo Fisher Scientific, MA, USA) spectrometer equipped with an $\mathrm{Al} \mathrm{K} \alpha$ X-ray source $(\mathrm{hv}=1486.6 \mathrm{eV})$, with a take-off angle of 0 degrees. The thermal decomposition behaviors of the materials were recorded on an STA 449C simultaneous DSC-TGA (Netzsch Instruments, Selb, Germany) thermal gravimetric analyzer (TGA) with a heating rate of $10^{\circ} \mathrm{C} / \mathrm{min}^{-1}$ in nitrogen atmosphere.

\subsection{Synthesis of $\mathrm{HS}-\mathrm{Br}$}

HS-Br was prepared via the esterification of acyl bromide groups in BIBB and hydroxyl groups in HS. The following procedure was adopted: $2 \mathrm{~g} \mathrm{SiO}_{2} \mathrm{HS}$ and $15 \mathrm{~mL}$ dichloromethane were added to a $100 \mathrm{~mL}$ reactor equipped with a magnetic stirrer. The mixture was stirring at room temperature for $10 \mathrm{~min}$. Subsequently, the solution of BIBB $(1.2 \mathrm{~mL}, 0.01 \mathrm{~mol})$ dissolved in $5 \mathrm{~mL}$ dichloromethane was dropped slowly into the round-bottom flask by a constant pressure liquid funnel. After reacting for $12 \mathrm{~h}$ at room temperature, the crude HS-Br was separated by centrifugation and washed completely by Soxhlet extraction with THF. The obtained HS-Br initiator was dried at $30{ }^{\circ} \mathrm{C}$ in vacuum. Elem. Anal. Calcd. (\%): C, 2.37; H, 3.4; $\mathrm{Br}, 0.473 \mathrm{mmol} \mathrm{g}^{-1}$ (calculated according to the $\mathrm{C}$ content) (Table 1).

Table 1. Elemental analysis of hollow spheres (HS), HS-Br, HS-1 (HS-g-PMMA), HS-2 (HS-g-PMMA-b-PNIPAM).

\begin{tabular}{ccccc}
\hline \multicolumn{1}{r}{ Sample } & Element/\% & N & C & H \\
\hline HS & 0 & 0.10 & 0.88 \\
HS-Br & 0 & 2.37 & 3.40 \\
HS-1 (HS- $g$-PMMA) & 0 & 6.38 & 1.34 \\
HS-2 (HS- $g$-PMMA- $b$-PNIPAM) & 1.24 & 10.01 & 1.81 \\
\hline
\end{tabular}

\subsection{Synthesis of HS-g-PMMA}

HS- $g$-PMMA was been synthesized through a new method of metal-free ATRP. In brief, HS-Br (0.15 g, $0.071 \mathrm{mmol} \mathrm{Br}$ groups), Methyl methacrylate (MMA) (1.6 mL, $15 \mathrm{mmol}), \mathrm{PTH}(0.0042 \mathrm{~g}$, $0.015 \mathrm{mmol})$, Ethyl 2-bromoisobutyrate $(5.5 \mu \mathrm{L}, 0.0375 \mathrm{mmol})$, and $1 \mathrm{~mL}$ DMA were mixed in a $10 \mathrm{~mL}$ quartz tube with a magnetic stirrer. The reaction mixture was stirred for $10 \mathrm{~min}$ in the dark at room temperature. When the reaction system was uniformly dispersed, the reaction system was irradiated under an ultraviolet lamp with $365 \mathrm{~nm}$ in the dark at room temperature; after reacting for $5 \mathrm{~h}$, the reaction mixture was separated by centrifugation. The obtained solids (crude HS-g-PMMA) were precipitated in methanol respectively and dried at $40{ }^{\circ} \mathrm{C}$ in vacuum. Afterwards, the HS- $g$-PMMA hybrid material was obtained by Soxhlet's extraction with THF for $12 \mathrm{~h}$ to remove the ungrafted PMMA completely and dried at $40^{\circ} \mathrm{C}$ in vacuum. The solution after centrifugation was also precipitated in methanol and dried to afford PMMA. Elem. Anal. Calcd. (\%): C, 6.38; H, 1.34; PMMA, $0.668 \mathrm{mmol} \mathrm{g}^{-1}$ (calculated according to the $\mathrm{C}$ content) (Table 1).

\subsection{Synthesis of HS-g-PMMA-b-PNIPAM}

HS- $g$-PMMA- $b$-PNIPAM was synthesized through a new method of metal-free ATRP using HS- $g$-PMMA as the macroinitiaor. In brief, $0.1 \mathrm{~g}$ HS- $g$-PMMA, NIPAM (0.42 g, $3.75 \mathrm{mmol})$, PTH $(0.001 \mathrm{~g}, 0.00375 \mathrm{mmol})$, Ethyl 2-bromoisobutyrate $(1.67 \mu \mathrm{L}, 0.0125 \mathrm{mmol})$, and $1 \mathrm{~mL}$ DMA were mixed in a $10 \mathrm{~mL}$ quartz tube with a magnetic stirrer. The reaction mixture was stirred for $10 \mathrm{~min}$ in the dark at room temperature. When the reaction system was uniformly dispersed, the reaction 
system was irradiated under an ultraviolet lamp with $365 \mathrm{~nm}$ in the dark at room temperature; after reacting for $5 \mathrm{~h}$, the reaction mixture was separated by centrifugation. The obtained solids (crude HS- $g$-PMMA- $b$-PNIPAM) were precipitated in methanol and dried at $40{ }^{\circ} \mathrm{C}$ in vacuum. Then, the crude HS- $g$-PMMA- $b$-PNIPAM was extracted with THF to remove the ungrafted polymers completely. The solution after centrifugation was precipitated in cold ether and resulted in PNIPAM. The detailed reaction process is listed in Scheme 1. Elem. Anal. Calcd. (\%): N, 1.24; C, 10.01; H, 1.81; PNIPAM, $0.504 \mathrm{mmol} \mathrm{g}^{-1}$ (calculated according to the $C$ content) (Table 1), so the molar ratio of PMMA and PNIPAM for HS- $g$-PMMA- $b$-PNIPAM is 1.32:1.

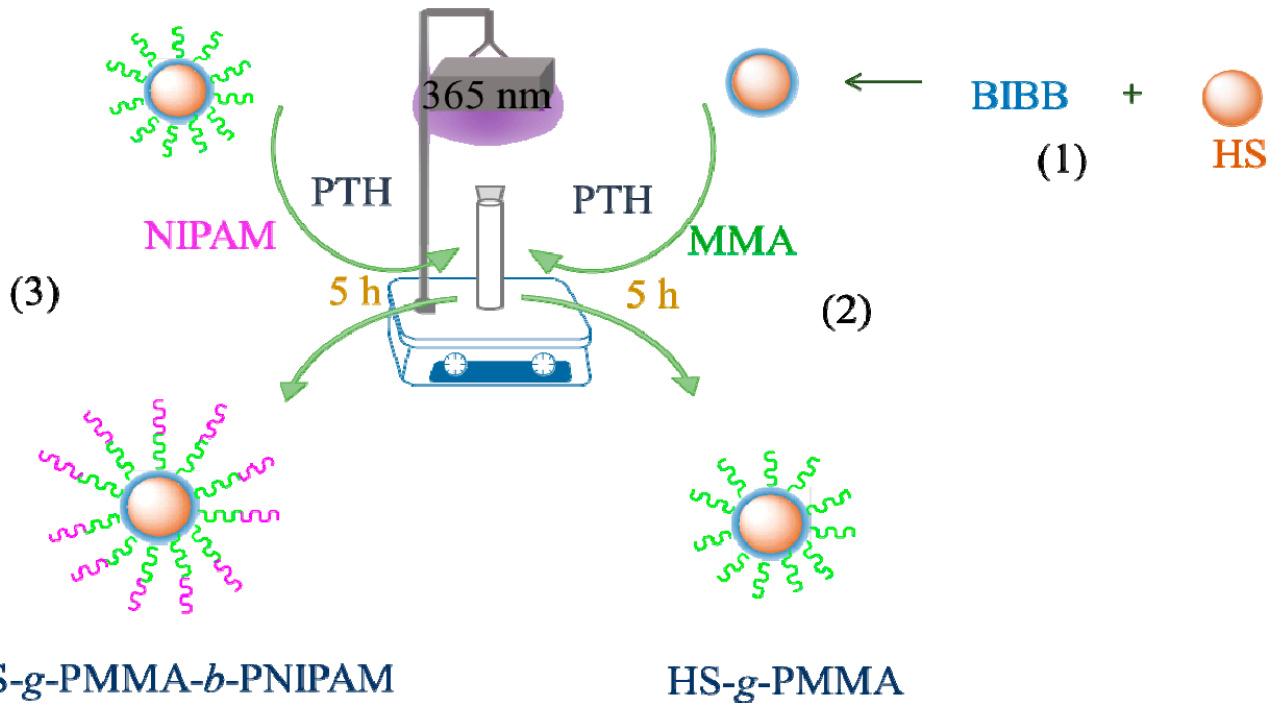

Scheme 1. The synthetic routes of HS- $g$-PMMA and HS- $g$-PMMA- $b$-PNIPAM.

\section{Results and Discussion}

\subsection{Structure Analysis}

HS-g-PMMA- $b$-PNIPAM was synthesized by metal-free surface-initiated ATRP (SI-ATRP). To prepare the copolymer brushes grafted from the HS surface via SI-ATRP, the immobilization of the ATRP initiator onto HS surfaces is necessary. The chemical compositions of HS and HS-Br were detected by XPS analysis. As shown in Figure 1, the surface of a HS is mainly composed by $\mathrm{Si}$ and O elements. Compared with crude HS, the HS-Br surfaces clearly show new $\mathrm{C}$ and $\mathrm{Br}$ elements. The peaks of $\mathrm{C} 1 \mathrm{~s}(284.5 \mathrm{eV})$ and $\mathrm{Br} 3 \mathrm{~d}(68.4 \mathrm{eV})$ are ascribed to the $\mathrm{C}$ and $\mathrm{Br}$ of BIBB on the HS surface. The $\mathrm{C} 1 \mathrm{~s}$ core-level spectra of the $\mathrm{HS}-\mathrm{Br}$ surfaces (Figure 1c) could be curve-fitted into five peak components, which were attributable to the $\mathrm{O}-\mathrm{C}=\mathrm{O}, \mathrm{C}=\mathrm{O}, \mathrm{C}-\mathrm{Br}, \mathrm{C}-\mathrm{C}$, and $\mathrm{C}-\mathrm{H}$ species of $\mathrm{HS}-\mathrm{Br}$, respectively. The observed signals for $\mathrm{Si}_{2 p}(102.1 \mathrm{eV})$ are attributed to exposed $\mathrm{Si}$ element of HS. The C, Br, and Si signals suggested that the ATRP initiator has been successfully immobilized onto HS surfaces, which provides the necessary conditions for the following metal-free surface-initiated ATRP. The element compositions (atomic \%) of C and Br in HS-Br based on XPS analysis are listed in Table S1. 
a
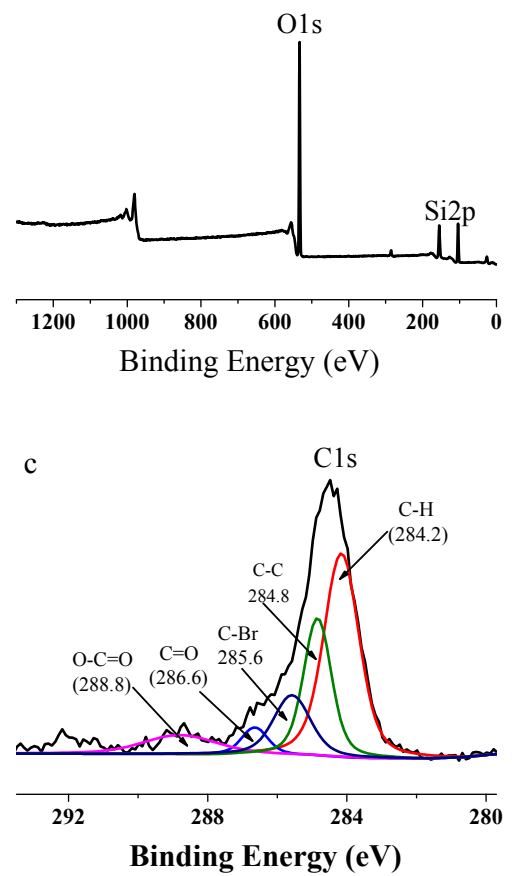
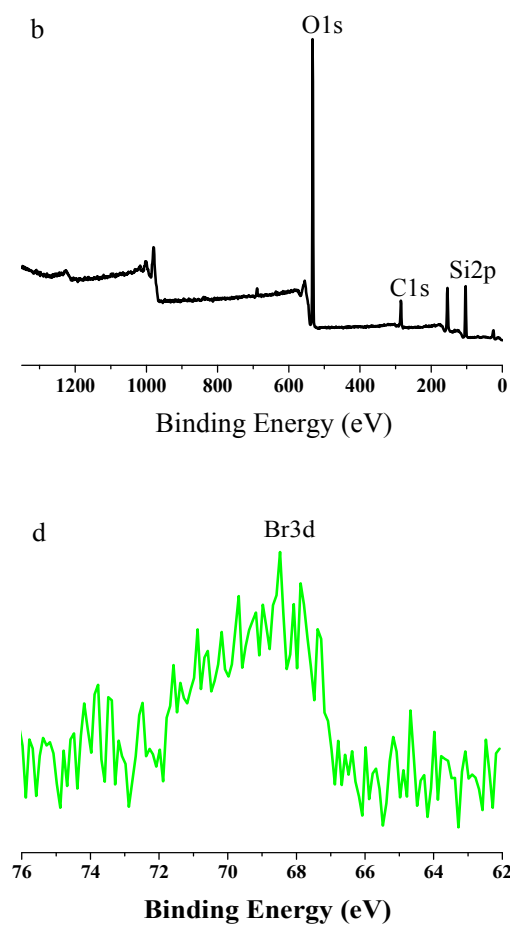

Figure 1. X-ray photoelectron spectroscopy (XPS) spectra of HS (a) and HS-Br (b-d): Survey scan curves range from 0 to $1350 \mathrm{eV}$ (b), the $\mathrm{C} 1$ s core-level spectra (c) and $\mathrm{Br} 3 \mathrm{~d}$ region (d).

Figure 2 shows the FT-IR spectra of the HS, HS-Br, HS- $g$-PMMA, and HS- $g$-PMMA- $b$-PNIPAM. The peaks at $1093 \mathrm{~cm}^{-1}$ are attributed to the stretching vibration absorption of Si-O-Si of HS. Compared with crude HS, the FT-IR spectra of HS-Br clearly shows a new characteristic peak at $1732 \mathrm{~cm}^{-1}$ which belongs to the $\mathrm{C}=\mathrm{O}$ stretching vibration of $\mathrm{BiBB}$ (Figure 2A,B), indicating the successful esterification of acyl bromide groups and hydroxyl groups. After metal-free SI-ATRP, the enhancive peak at $1732 \mathrm{~cm}^{-1}$ belongs to the $\mathrm{C}=\mathrm{O}$ stretching vibration which is the result of the $\mathrm{C}=\mathrm{O}$ overlap of BiBB and PMMA. As for the HS- $g$-PMMA- $b$-PNIPAM, the absorption peak at $1654 \mathrm{~cm}^{-1}$ accords with the $-\mathrm{CO}-\mathrm{N}$ - stretching vibration of PNIPAM, and the decreased peak at $1732 \mathrm{~cm}^{-1}$ resulted from the $\mathrm{C}=\mathrm{O}$ stretching vibration of PMMA, which indicated the successful grafting of PMMA and PNIPAM onto HS surfaces.

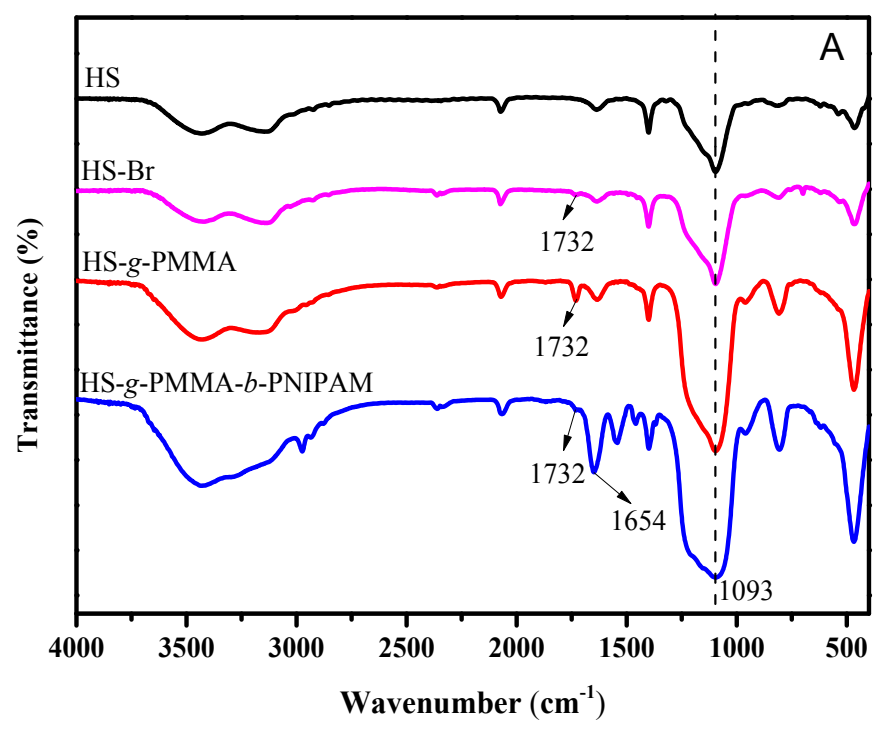

Figure 2. Cont. 


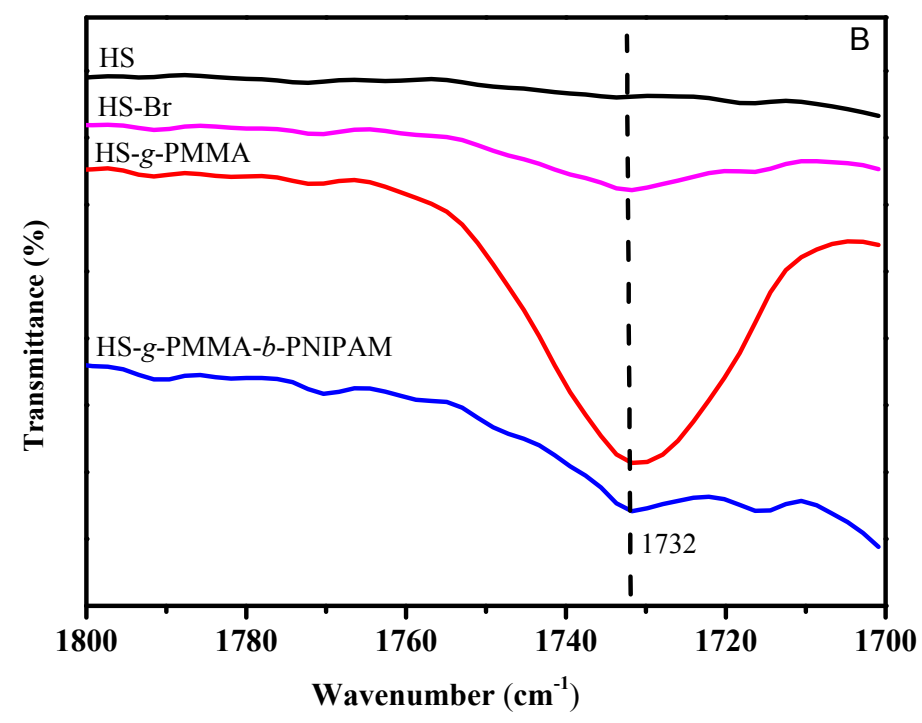

Figure 2. Fourier transform infrared spectroscopy (FT-IR) spectra (A) and magnified FT-IR spectra in the region $1700-1800 \mathrm{~cm}^{-1}$ (B) of hollow spheres, HS-Br, HS- $g$-PMMA, and HS-g-PMMA- $b$-PNIPAM.

The ${ }^{1} \mathrm{H}$ NMR spectrum of PMMA formed in solution also gives support to the metal-free SI-ATRP mechanism (Figure 3). Except for the peak of NMR internal reference (TMS) at $0.00 \mathrm{ppm}$ and solvent $\left(\mathrm{CDCl}_{3}\right)$ at $7.27 \mathrm{ppm}$, the signals at around $1.81 \mathrm{ppm}$ and $0.80-1.02 \mathrm{ppm}$ are ascribed to the protons of methylene and methyl to the repeat unit in the main polymer chain. The peak at $3.78 \mathrm{ppm}$ is attributed to the methyl ester group $\left(-\mathrm{OCH}_{3}\right)$ at the chain end in PMMA, as mentioned by Zhang [19], which deviated from the chemical shift $(3.60 \mathrm{ppm})$ of the other $-\mathrm{OCH}_{3}$ due to the electron attracting function of the $\mathrm{Br}$ atom at the chain end. The ${ }^{1} \mathrm{H}$ NMR spectrum result indicated that the $\mathrm{Br}$ atom was located at the end of the main PMMA polymer chain.

Figure 4 show the GPC traces of PMMA and PNIPAM formed in solution. The molecular weight (Mn) values reached 53,400 and 30,700 g/mol, respectively, and the molecular weight distributions $(\mathrm{Mw} / \mathrm{Mn})$ were narrow (1.33 and 1.41, respectively), which indicated that the polymerization process possesses the characteristic of controlled/ "living" polymerization.

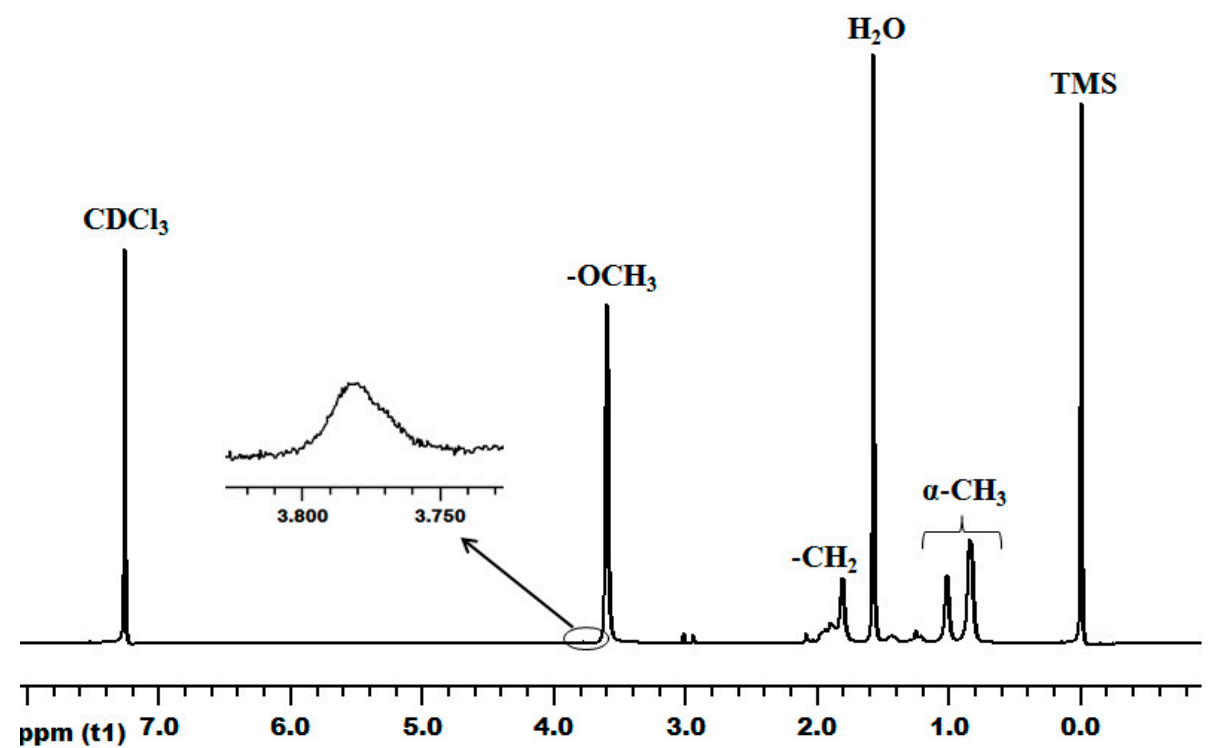

Figure 3. ${ }^{1} \mathrm{H}$-nuclear magnetic resonance spectroscopy $\left({ }^{1} \mathrm{H}\right.$ NMR) spectrum of PMMA formed in solution. 


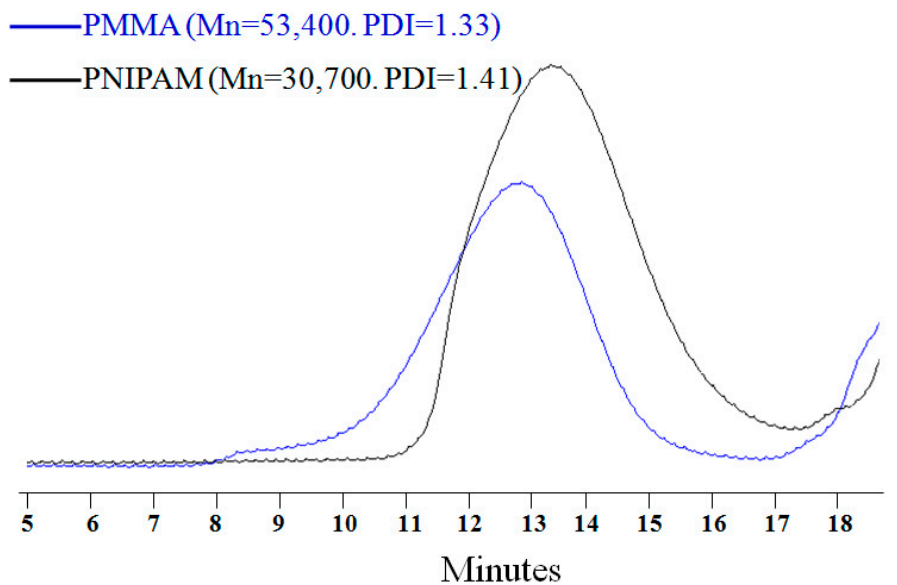

Figure 4. Gel permeation chromatography (GPC) traces of PMMA and PNIPAM.

\subsection{Morphology Analysis}

In order to obtain more information on the effects of polymer grafting onto the microstructures of hollow spheres, TEM was used to investigate the surface topography in detail. Figure 5 shows the TEM images of hollow spheres, HS- $g$-PMMA, and HS- $g$-PMMA- $b$-PNIPAM. The hollow nature of the hollow spheres was observed by the contrast in TEM observations, as shown in Figure 5, and it can be observed that the diameter of the wall thickness exhibits a tendency to increase with process of grafting polymerization. The average diameter of the hollow spheres mainly centered at $1.2-1.3 \mu \mathrm{m}$, and the average wall thickness mainly focused on 90-110 nm before grafting polymerization, as indicated by the size distribution histogram (Figure 5a). After grafting polymerization (Figure 5b,c), a small number of hollow spheres were broken (Figure $5 c$ ), and the average wall thickness of the hollow spheres increased from 100 to 105 and $115 \mathrm{~nm}$, respectively, owing to the grafting polymerization of MMA and PNIPAM on the surfaces of hollow spheres.

(a)
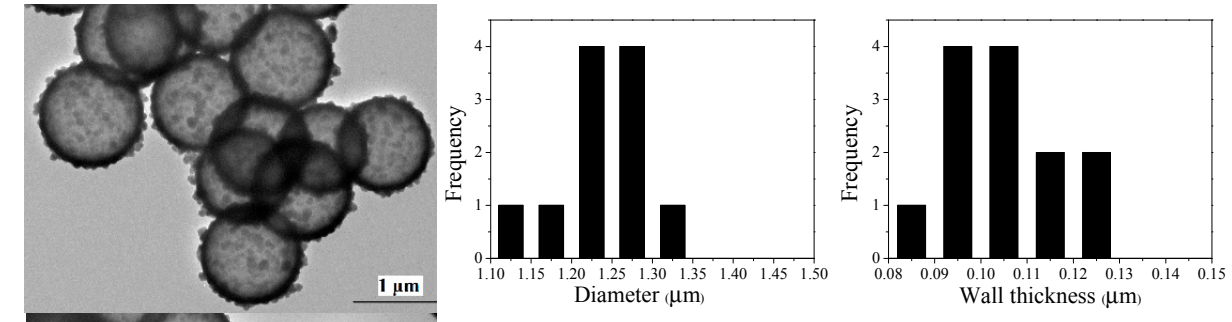

(b)
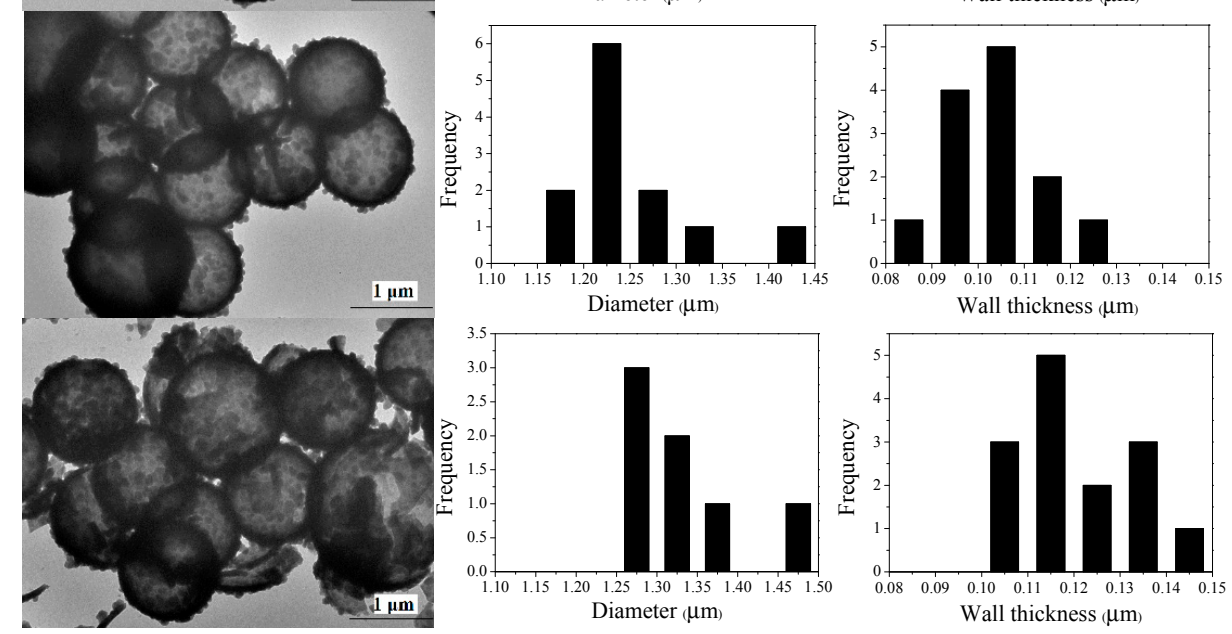

Diameter $(\mu \mathrm{m})$
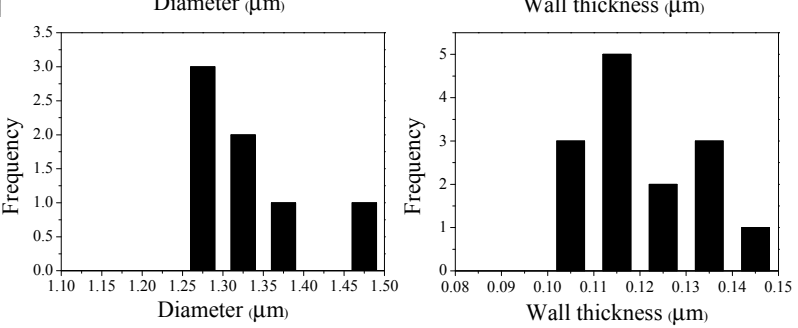

Figure 5. Transmission electron microscope (TEM) images of (a) hollow spheres, (b) HS-g-PMMA, and (c) HS- $g$-PMMA- $b$-PNIPAM. 


\subsection{TGA Analysis}

The TGA was used to determine the grafting percentage of polymers on the surface of hollow spheres and investigate the thermostability of hollow sphere samples. Figure 6 shows the TGA curves of hollow spheres, HS- $g$-PMMA, and HS- $g$-PMMA- $b$-PNIPAM with temperature increasing from room temperature to $800^{\circ} \mathrm{C}$. The weight loss experienced by the hollow spheres, HS-g-PMMA, and HS- $g$-PMMA- $b$-PNIPAM before $100{ }^{\circ} \mathrm{C}$ is attributed to the loss of adsorbed water in the samples (zone I). The second significant weight loss was observed in the range of $100-420{ }^{\circ} \mathrm{C}$ (zone II), which is due to the thermal decomposition of the organic content in PMMA and PNIPAM (the thermal decomposition temperature of pure PMMA and pure PNIPAM is $420{ }^{\circ} \mathrm{C}$, Figure $\left.6 \mathrm{e}, \mathrm{f}\right)$. The third weight loss zone is associated with the inorganic salts remaining in the hollow spheres (zone III). The pure hollow spheres show no obvious decomposition (3.7\%) between room temperature and $800{ }^{\circ} \mathrm{C}$. Therefore, the grafting percentage of polymers on the surfaces of the hollow spheres can be obtained via the weight loss of zone II. The organic content calculated for HS-g-PMMA is $7.87 \%$. Compared with the HS- $g$-PMMA, the calculated organic content of PMMA- $b$-PNIPAM increased to $11.49 \%$. That is, the grafted PNIPAM on hollow spheres surface is about $3.62 \%$. This indicated that both PMMA and PNIPAM have successfully grafted onto the hollow sphere surfaces.
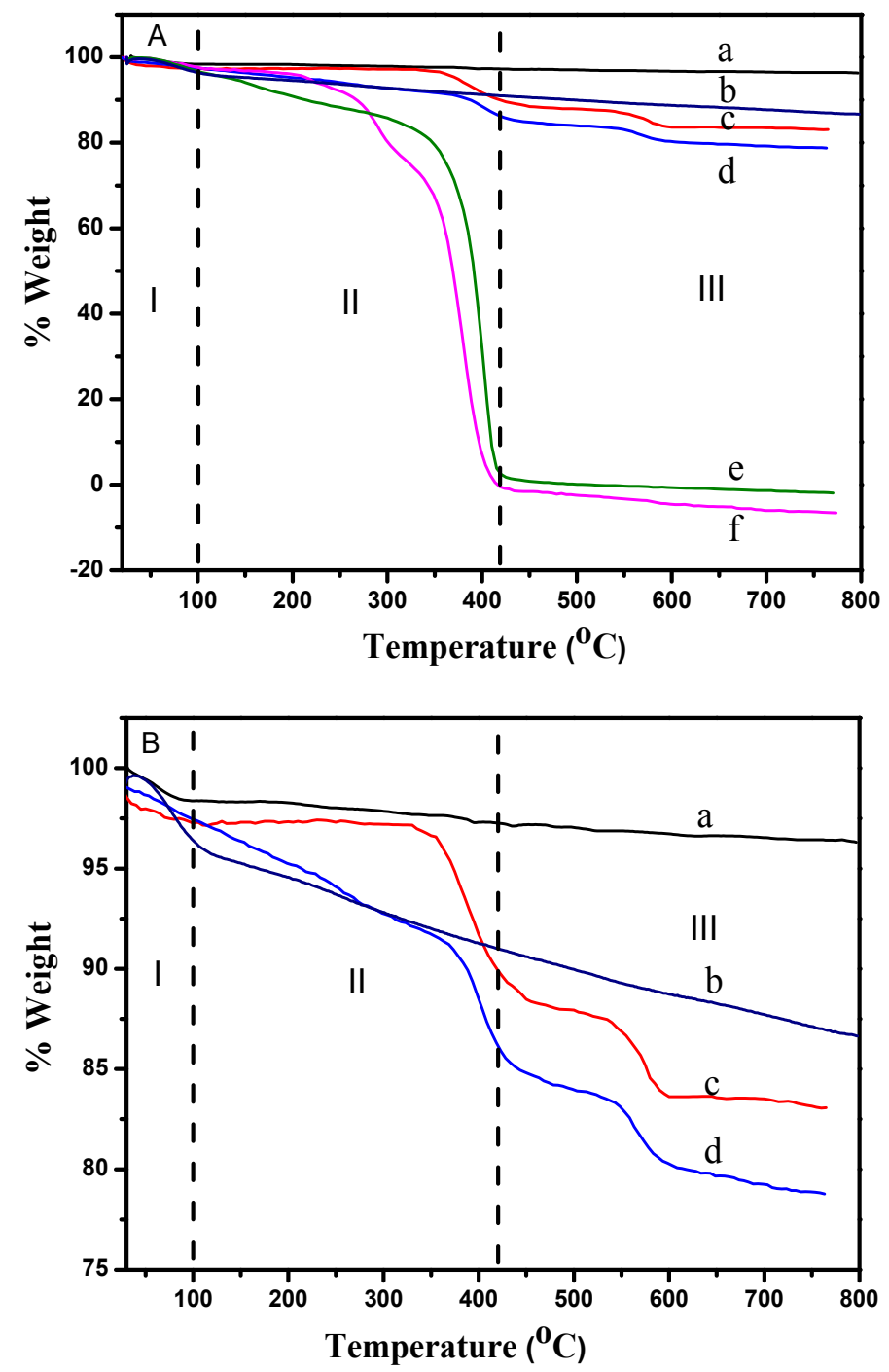

Figure 6. (A) TG curves of (a) hollow spheres, (b) HS-Br, (c) HS- $g$-PMMA, (d) HS- $g$-PMMA- $b$-PNIPAM, (e) pure PMMA and (f) pure PNIPAM. (B) Magnified TG curves of (a) hollow spheres, (b) HS-Br, (c) HS- $g$-PMMA, and (d) HS- $g$-PMMA- $b$-PNIPAM. 


\subsection{Dispersibility in Solvents}

Figure 7 presents the images of the dispersions of hollow spheres samples in inorganic $\left(\mathrm{H}_{2} \mathrm{O}\right)$ and organic solvent (THF). The pure HS are dispersed in water and aggregated in THF; the grafting of PMMA onto HS surfaces (HS-1 (HS- $g$-PMMA)) resulted in the complete opposite dispersibility. They are aggregated in the aqueous phase but readily dispersed in THF. These results show that the grafted PMMA contributed to the surface hydrophobization of the hollow spheres. The surface modification of HS with PMMA- $b$-PNIPAM (HS-2 (HS- $g$-PMMA- $b$-PNIPAM)) alters the dispersion again. The PNIPAM chains were shown to contribute to increasing the surface amphiphilicity of HS.

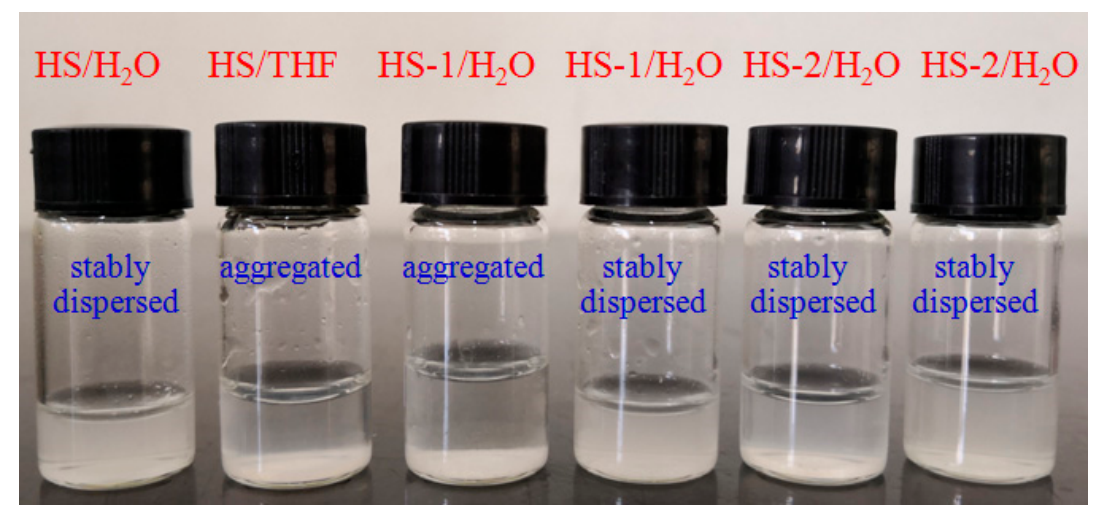

Figure 7. Dispersed/aggregated state of the hollow spheres samples in $\mathrm{H}_{2} \mathrm{O}$ /THF, respectively.

\section{Conclusions}

In summary, we reported a facile synthesis of well-defined amphiphilic diblock copolymer grafted hollow spheres via metal-free SI-ATRP. The ATRP initiators $\alpha$-Bromoisobutyryl bromide (BIBB) were attached onto the surfaces of hollow spheres to initiate metal-free SI-ATRP of MMA and NIPAM. It was found that the BIBB had been immobilized onto HS surfaces successfully. Characterization with FT-IR, ${ }^{1} \mathrm{HNMR}$, TEM, and TGA verified the success of the metal-free SI-ATRP of MMA, and the Br atom had located at the end of the main PMMA polymer chain, which gives HS-g-PMMA the ability to extend the chains by sequential addition of NIPAM. Furthermore, the molecular weight distributions $(\mathrm{Mw} / \mathrm{Mn})$ of PMMA and PNIPAM formed in solution is narrow (1.33 and 1.41, respectively), which indicated that the polymerizations process possesses the characteristic of controlled/"living" polymerization.

Supplementary Materials: The following are available online at http:/ /www.mdpi.com/2073-4360/11/4/599/s1, Figure S1: ${ }^{1} \mathrm{H}$ NMR spectrum of 10-phenylphenothiazine (PTH), Table S1: Element compositions (atomic \%) of HS-Br based on XPS analysis.

Author Contributions: C.-N.Y., Q.L., L.X., and L.-P.B. performed the experiments, analyzed the data, and wrote the paper; L.-P.W. conceived and designed the experiments and revised the paper; G.L. provided the overall consulting.

Funding: This research was funded by Shandong Province Higher Education Science and Technology Program (J16LA01), the Natural Science Foundation of China (No. 51503094), Natural Science Foundation of Shandong Province (ZR2015BQ002), and the Science and Technology Innovation Fund of Liaocheng University $(26312171917,26312171930)$.

Acknowledgments: This research was supported by the financial support from the Shandong Province Higher Education Science and Technology Program (J16LA01), the Natural Science Foundation of China (No. 51503094), Natural Science Foundation of Shandong Province (ZR2015BQ002), and the Science and Technology Innovation Fund of Liaocheng University (26312171917, 26312171930).

Conflicts of Interest: The authors declare no conflict of interest.

\section{References}

1. Yan, J.; Pan, X.; Wang, Z.; Lu, Z.; Wang, Y.; Liu, L.; Zhang, J.; Ho, C.; Bockstaller, M.R.; Matyjaszewski, K. A fatty acid-inspired tetherable initiator for surface-initiated atom transfer radical polymerization. Chem. Mater. 2017, 29, 4963-4969. [CrossRef] 
2. Liu, X.; Appelhans, D.; Zhang, T.; Voit, B. Rapid synthesis of dual-responsive hollow capsules with controllable membrane thickness by surface-initiated SET-LRP polymerization. Macromolecules 2018, 51, 1011-1019. [CrossRef]

3. Mrlik, M.; Cvek, M.; Osicka, J.; Moucka, R.; Sedlacik, M.; Pavlinek, V. Surface-initiated atom transfer radical polymerization from graphene oxide: A way towards fine tuning of electric conductivity and electroresponsive capabilities. Mater. Lett. 2018, 211, 138-141. [CrossRef]

4. Su, J.; He, X.; Chen, L.; Zhang, Y. A combination of "thiol-ene" click chemistry and surface initiated atom transfer radical polymerization: Fabrication of boronic acid functionalized magnetic graphene oxide composite for enrichment of glycoproteins. Talanta 2018, 180, 54-60. [CrossRef] [PubMed]

5. Wang, L.P.; Li, G.; Li, W.Z.; Ban, C.L.; Li, Y.C.; Guo, C.; Zhang, M.M.; Liu, L.Y.; Lu, N.N.; Zheng, M.Z. Copolymers with fluorescence properties in mesoporous silica SBA-15:Synthesis and characterization. Chin. Chem. Lett. 2014, 25, 1620-1624. [CrossRef]

6. Pan, X.; Fantin, M.; Yuan, F.; Matyjaszewski, K. Externally controlled atom transfer radical polymerization. Chem. Soc. Rev. 2018, 47, 5457-5490. [CrossRef] [PubMed]

7. Kopeć, M.; Spanjers, J.; Scavo, E.; Ernens, D.; Duvigneau, J.; Vancso, G.J. Surface-initiated ATRP from polydopamine-modified $\mathrm{TiO}_{2}$ nanoparticles. Eur. Polym. J. 2018, 106, 291-296. [CrossRef]

8. Lu, C.; Yu, J.; Wang, C.; Wang, J.; Chu, F. Fabrication of UV-absorbent cellulose-rosin based thermoplastic elastomer via "graft from" ATRP. Carbohydr. Polym. 2018, 188, 128-135. [CrossRef]

9. Wilson, O.R.; Magenau, A.J.D. Oxygen tolerant and room temperature RAFT through alkylborane initiation. ACS Macro Lett. 2018, 7, 370-375. [CrossRef]

10. Shen, L.; Lu, Q.; Zhu, A.; Lv, X.; An, Z. Photocontrolled RAFT polymerization mediated by a supramolecular catalyst. ACS Macro. Lett. 2017, 6, 625-631. [CrossRef]

11. Ishigaki, Y.; Mori, H. Synthesis of poly(chloroprene)-based block copolymers by RAFT mediated emulsion polymerization. Polymer 2018, 140, 198-207. [CrossRef]

12. Boyer, C.; Lacroix-Desmazes, P.; Robin, J.J.; Boutevin, B. Reverse iodine transfer polymerization (RITP) of methyl methacrylate. Macromolecules 2006, 39, 4044-4053. [CrossRef]

13. Tonnar, J.; Lacroix-Desmazes, P.; Boutevin, B. Living radical ab initio emulsion polymerization of n-butyl acrylate by reverse iodine transfer polymerization (RITP): use of persulfate as both initiator and oxidant. Macromolecules 2007, 40, 6076-6081. [CrossRef]

14. Wang, L.P.; Li, Y.C.; Chen, L.F.; Ban, C.L.; Li, G.; Ni, J.J. Fabrication of honeycomb-patterned porous films from PS-b-PNIPAM amphiphilic diblock copolymers synthesized via RITP. J. Colloid Interface Sci. 2014, 420, 112-118. [CrossRef]

15. Oikonomou, E.K.; Pefkianakis, E.K.; Bokias, G.; Kallitsis, J.K. Direct synthesis of amphiphilic block copolymers, consisting of poly(methyl methacrylate) and poly(sodium styrene sulfonate) blocks through atom transfer radical polymerization. Eur. Polym. J. 2008, 44, 1857-1864. [CrossRef]

16. Oikonomou, E.K.; Bokias, G.; Iliopoulos, I.; Kallitsis, J.K. Sequential association of anionic/thermosensitive diblock copolymers with cationic surfactants. Macromolecules 2013, 46, 1082-1092. [CrossRef]

17. Huang, Y.; Sasano, T.; Tsujii, Y.; Ohno, K. Well-Defined Polymer-Brush-Coated Rod-Shaped Particles: Synthesis and Formation of Liquid Crystals. Macromolecules 2016, 49, 8430-8439. [CrossRef]

18. Ohno, K.; Mori, C.; Akashi, T.; Yoshida, S.; Tago, Y.; Tsujii, Y.; Tabata, Y. Fabrication of Contrast Agents for Magnetic Resonance Imaging from Polymer-Brush-Afforded Iron Oxide Magnetic Nanoparticles Prepared by Surface-Initiated Living Radical Polymerization. Biomacromolecules 2013, 14, 3453-3462. [CrossRef]

19. Chen, M.; Zhou, H.; Zhou, L.; Zhang, F. Confined polymerization: ARGET ATRP of MMA in the nanopores of modified SBA-15. Polymer 2017, 114, 180-188. [CrossRef]

20. Kaßel, M.; Gerke, J.; Ley, A.; Vana, P. Surface modification of wood flour via ARGET ATRP and its application as filler in thermoplastics. Polymers 2018, 10, 354. [CrossRef]

21. Cordero, R.; Jawaid, A.; Hsiao, M.S.; Lequeux, Z.; Vaia, R.A.; Ober, C.K. Mini monomer encapsulated emulsion polymerization of PMMA using aqueous ARGET ATRP. ACS Macro Lett. 2018, 7, 459-463. [CrossRef]

22. Su, X.; Nishizawa, K.; Bultz, E.; Sawamoto, M.; Ouchi, M.; Jessop, P.G.; Cunningham, M.F. Living $\mathrm{CO}_{2}$-switchable latexes prepared via emulsion ATRP and AGET miniemulsion ATRP. Macromolecules 2016, 49, 6251-6259. [CrossRef] 
23. Simakova, A.; Averick, S.E.; Konkolewicz, D.; Matyjaszewski, K. Aqueous ARGET ATRP. Macromolecules 2012, 45, 6371-6379. [CrossRef]

24. Ribelli, T.G.; Fantin, M.; Daran, J.C.; Augustine, K.F.; Poli, R.; Matyjaszewski, K. Synthesis and characterization of the most active copper ATRP catalyst based on tris[(4-dimethylaminopyridyl)methyl]amine. J. Am. Chem. Soc. 2018, 140, 1525-1534. [CrossRef]

25. Fu, L.; Simakova, A.; Fantin, M.; Wang, Y.; Matyjaszewski, K. Direct ATRP of methacrylic acid with iron-porphyrin based catalysts. ACS Macro Lett. 2018, 7, 26-30. [CrossRef] [PubMed]

26. Wang, Y.; Lorandi, F.; Fantin, M.; Chmielarz, P.; Isse, A.A.; Gennaro, A.; Matyjaszewski, K. Miniemulsion ARGET ATRP via interfacial and ion-pair catalysis: From ppm to ppb of residual copper. Macromolecules 2017, 50, 8417-8425. [CrossRef]

27. Chmielarz, P.; Fantin, M.; Park, S.; Isse, A.A.; Gennaro, A.; Magenau, A.J.D.; Sobkowiak, A.; Matyjaszewski, K. Electrochemically mediated atom transfer radical polymerization (eATRP). Prog. Polym. Sci. 2017, 69, 47-78. [CrossRef]

28. Yan, J.; Pan, X.; Schmitt, M.; Wang, Z.; Bockstaller, M.R.; Matyjaszewski, K. Enhancing initiation efficiency in metal-free surface-initiated atom transfer radical polymerization (SI-ATRP). ACS Macro Lett. 2016, 5, 661-665. [CrossRef]

29. Liu, X.; Zhang, L.; Cheng, Z.; Zhu, X. Metal-free photoinduced electron transfer-atom transfer radical polymerization (PET-ATRP) via a visible light organic photocatalyst. Polym. Chem. 2016, 7, 689-700. [CrossRef]

30. Treat, N.J.; Sprafke, H.; Kramer, J.W.; Clark, P.G.; Barton, B.E.; Alaniz, J.R.; Fors, B.P.; Hawker, C.J. Metal-free atom transfer radical polymerization. J. Am. Chem. Soc. 2014, 136, 16096-16101. [CrossRef]

31. Aydogan, C.; Yilmaz, G.; Yagci, Y. Synthesis of hyperbranched polymers by photoinduced metal-free ATRP. Macromolecules 2017, 50, 9115-9120. [CrossRef]

32. Kutahya, C.; Allushi, A.; Isci, R.; Kreutzer, J.; Ozturk, T.; Yilmaz, G.; Yagci, Y. Photoinduced metal-free atom transfer radical polymerization using highly conjugated thienothiophene derivatives. Macromolecules 2017, 50, 6903-6910. [CrossRef]

33. Wang, J.; Yuan, L.; Wang, Z.; Rahman, M.A.; Huang, Y.; Zhu, T.; Wang, R.; Cheng, J.; Wang, C.; Chu, F.; et al. Photoinduced metal-free atom transfer radical polymerization of biomass-based monomers. Macromolecules 2016, 49, 7709-7717. [CrossRef]

34. Pan, X.; Fang, C.; Fantin, M.; Malhotra, N.; So, W.Y.; Peteanu, L.A.; Isse, A.A.; Gennaro, A.; Liu, P.; Matyjaszewski, K. Mechanism of photoinduced metal-free atom transfer radical polymerization: Experimental and computational studies. J. Am. Chem. Soc. 2016, 138, 2411-2425. [CrossRef]

35. Pan, X.; Lamson, M.; Yan, J.; Matyjaszewski, K. Photoinduced metal-free atom transfer radical polymerization of acrylonitrile. ACS Macro Lett. 2015, 4, 192-196. [CrossRef]

36. Ma, L.; Li, N.; Zhu, J.; Chen, X. Visible Light-Induced Metal Free Surface Initiated Atom Transfer Radical Polymerization of Methyl Methacrylate on SBA-15. Polymers 2017, 9, 58. [CrossRef]

37. Zeng, G.; Liu, M.; Shi, K.; Heng, C.; Mao, L.; Wan, Q.; Huang, H.; Deng, F.; Zhang, X.; Wei, Y. Surface modification of nanodiamond through metal free atomtransfer radical polymerization. Appl. Surf. Sci. 2016, 390, 710-717. [CrossRef]

38. Zeng, G.; Liu, M.; Heng, C.; Huang, Q.; Mao, L.; Huang, H.; Hui, J.; Deng, F.; Zhang, X.; Wei, Y. Surface polyPEGylation of $\mathrm{Eu}^{3+}$ doped luminescent hydroxyapatite nanorods through the combination of ligand exchange and metal free surface initiated atom transfer radical polymerization. Appl. Surf. Sci. 2017, 399, 499-505. [CrossRef]

39. Fang, M.; Chen, Z.; Tian, Q.; Cao, Y.; Wang, C.; Liu, Y.; Fu, J.; Zhang, J.; Zhu, L.; Yang, C.; et al. Synthesis of uniform discrete cage-like nitrogen-doped hollow porous carbon spheres with tunable direct large mesoporous for ultrahigh supercapacitive performance. Appl. Surf. Sci. 2017, 425, 69-76. [CrossRef]

40. Liu, X.; Chen, Y.; Liu, H.; Liu, Z.Q. $\mathrm{SiO}_{2} @ \mathrm{C}$ hollow sphere anodes for lithium-ion batteries. J. Mater. Sci. Technol. 2017, 33, 239-245. [CrossRef]

41. Takai-Yamashita, C.; Ishino, T.; Fuji, M.; Inoue, K. Preparation and formation mechanism of ZnO supported hollow $\mathrm{SiO}_{2}$ nanoparticle by an interfacial reaction through micropores. Colloids Surf. A 2016, 493, 9-17. [CrossRef]

42. Prier, C.K.; Rankic, D.A.; MacMillan, D.W.C. Visible light photoredox catalysis with transition metal complexes: Applications in organic synthesis. Chem. Rev. 2013, 113, 5322-5363. [CrossRef] [PubMed] 
43. Kim, M.K.; Kim, D.W.; Moon, S.H.; Shin, D.W.; Oh, T.S.; Yoo, J.B. One step process of decomposition and polymerization to fabricate $\mathrm{SiO}_{2}$ hollow spheres/polyimide composite for foldable OLEDs. Mater. Sci. Eng. B 2017, 217, 7-11. [CrossRef]

44. Šoltys, M.; Balouch, M.; Kašpar, O.; Lhotka, M.; Ulbrich, P.; Zadražil, A.; Kovačík, P.; Štěpánek, F. Evaluation of scale-up strategies for the batch synthesis of dense and hollow mesoporous silica microspheres. Chem. Eng. J. 2018, 334, 1135-1147. [CrossRef]

45. Chen, W.; Takai, C.; Khosroshahi, H.R.; Fuji, M.; Shirai, T. $\mathrm{SiO}_{2} / \mathrm{TiO}_{2}$ double-shell hollow particles: Fabrication and UV-Vis spectrum characterization. Adv. Powder Technol. 2016, 27, 812-818. [CrossRef]

46. Gao, R.; Chen, M.; Li, W.; Zhou, S.; Wu, L. Facile fabrication and some specific properties of polymeric/inorganic bilayer hybrid hollow spheres. J. Mater. Chem. A 2013, 1, 2183-2191. [CrossRef]

47. Wang, L.P.; Dong, L.H.; Hao, J.C.; Lv, X.H.; Li, W.Z.; Li, Y.C.; Zhen, J.M.; Hao, Y.C.; Ma, F. Fabrication of block copolymer brushes on hollow sphere surface via reverse iodine transfer polymerization. J. Colloid Interface Sci. 2011, 361, 400-406. [CrossRef]

48. Chang, F.; Huang, X.; Wei, H.; Chen, K.; Shan, C.; Tang, X. Intrinsically fluorescent hollow spheres based on organic-inorganic hybrid polyphosphazene material: Synthesis and application in drug release. Mater. Lett. 2014, 125, 128-131. [CrossRef]

49. Chen, M.; Wu, L.; Zhou, S.; You, B. A method for the fabrication of monodisperse hollow silica spheres. Adv. Mater. 2006, 18, 801-806. [CrossRef]

(C) 2019 by the authors. Licensee MDPI, Basel, Switzerland. This article is an open access article distributed under the terms and conditions of the Creative Commons Attribution (CC BY) license (http://creativecommons.org/licenses/by/4.0/). 